\title{
¿POR QUÉ “INFORMÁTICA JURÍDICA” HOY (2018)?
}

\section{${ }^{1}$ Fernando Galindo \\ ${ }^{2}$ Pilar Lasala}

\section{RESUMEN}

El trabajo plantea que no cabe pensar en 2018 que la expresión "informática jurídica" existe porque hay una disciplina académica que tiene el mismo nombre, sino que se utiliza porque es el modo de expresar la satisfacción de necesidades sociales relacionadas con el uso de las tecnologías de la información y la comunicación por medio del ejercicio conjunto, interdisciplinar, ante problemas concretos, de competencias profesionales propias de juristas y de ingenieros informáticos.

Palabras clave.- Dogmática; Competencias; Informática jurídica; jurista; ingeniero informático.

\section{WHY "LEGAL INFORMATICS" TODAY (2018)?}

\begin{abstract}
The paper suggests that it is not possible to think in 2018 that the expression "legal informatics" exists because there is an academic discipline with the same name, but it is used because it is the way of expressing the satisfaction of social needs related to the use of information and communication technologies by means of a joint and interdisciplinary exercise of professional competences of lawyers and computer engineers, in the face of specific problems.
\end{abstract}

Key words.- Dogmatics; Competences; Legal informatics; jurist; computer engineer.

\footnotetext{
${ }^{1}$ Catedrático de Filosofía del Derecho, Universidad de Zaragoza. cfa@unizar.es

${ }^{2}$ Profesora Titular de Estadística e Investigación Operativa, Universidad de Zaragoza. lasala@unizar.es
} 


\section{Introducción}

Aquí no se quiere hacer una fundamentación histórica sobre la expresión "informática jurídica" ${ }^{3}$. Basta decir que se utiliza desde hace años, y que cuenta con numerosas referencias bibliográficas en diferentes idiomas como "informática jurídica", "legal informatics", "informatique juridique" o "Rechtsinformatik" 4 . Resume lo anterior mencionar que estas expresiones surgieron cuando se constató una necesidad: la de regular un Derecho dirigido a considerar los efectos de la introducción de los programas de ordenador por las actividades humanas. Aquí a lo que se quiere dar respuesta es a otra cuestión: a la pregunta ¿por qué hablar sobre "informática jurídica” en 2018 en un ámbito de discusión de carácter jurídico?

La justificación de la pregunta reside en observar si existen razones para tratar sobre la vigencia de la expresión, tengan o no relación con las que estaban detrás de su origen, desarrollo y mantenimiento en el pasado.

A estos efectos interesa señalar que, como lo muestran los numerosos manuales de introducción a la materia editados en el momento de la aparación del término (segunda mitad de siglo XX), la más significativa última razón de su defensa en el pasado residía en la consideración de que la expresión "informática jurídica” podía tomarse como un nuevo objeto de estudio, una nueva ciencia, por parte de la reflexión jurídica: otra ciencia del Derecho. Dichos manuales tenían en general el objetivo de que su contenido fuera objeto de aprendizaje por los estudiantes de Derecho, como una nueva asignatura que debía contar con dicha denominación tal y como desde hace tiempo se hace con otras materias como Derecho civil o Derecho penal ${ }^{5}$.

Aparentemente la aspiración está próxima a ser una realidad desde la siguiente perspectiva: existen numerosos manuales que la desarrollan, también revistas científicas y trabajos o artículos que la consideran objeto de su reflexión, lo que explicaría su hipotética

\footnotetext{
${ }^{3}$ Las propuestas doctrinales recogidas en (PALIWALA, 2010), tienen gran interés a estos efectos al hacerse en la obra un balance histórico y recopilar posiciones de significativos autores que se han ocupado en varios países del estudio de la materia desde sus comienzos.

${ }^{4}$ Para refencias bibliográficas consultar: ibidem: pp. 255-285.

${ }^{5}$ Los manuales perseguían, además, satisfacer dos "mitos": el de la razón, con el que se ordena el conocimiento científico desde el Renacimiento, y el de la tecnología, mito que se hace cada vez más dominante en los siglos XX y XXI. Sobre los mitos (deidad, razón, estado, mercado y tecnología) y su defectuosa guía al conocimiento y la práctica, en especial en el ámbito jurídico versa la obra: (GRANT, BENNET MOSSES, 2017). Son de especial interés por el esclarecimiento del tema las páginas comprendidas entre la 1 y la 31.
} 
aceptación como estudio académico ${ }^{6}$. Pese a lo anterior la verdad es que la "informática jurídica" en escasa medida ha sido considerada como asignatura en los planes de estudio universitarios que se imparten hoy en Facultades de Derecho. Especialmente como materia con carácter obligatorio. La materia sigue en activo académicamente en algunas ocasiones con carácter optativo o, a lo sumo, como curso de especialización, pero no tiene la general aceptación que mantienen desde el siglo XIX las denominaciones jurídicas tradicionales o dogmáticas.

¿Qué hay detrás de estos hechos? De ello nos ocupamos en este trabajo fijándonos en lo siguiente.

En primer lugar (Capítulo 2) consideramos, brevemente, que pese a los cambios sociales producidos desde que se establecieron los Códigos racionales, consecuencia de la Ilustración del siglo XVIII, la docencia que prepara a juristas en las Facultades de Derecho ha cambiado en escasa medida: sigue centrándose básicamente en la explicación de los conceptos jurídicos, ordenados por la dogmática o la Ciencia del Derecho, gracias a los que se articula y transfiere el conocimiento del Derecho positivo, y ello pese a las fuertes críticas que se ha producido a este tipo de docencia desde prácticamente los principios del siglo XX por su marcado alejamiento de la práctica profesional.

En segundo lugar (Capítulo 3) mostramos frente a lo anterior la paradoja de que desde el comienzo de los 90, atendiendo a las indicaciones que en Europa se hace a la formación universitaria en general y a la de los juristas en concreto por los Ministros de Educación. Desde entonces la exigencia a la formación jurídica no es únicamente la de la formación en las distintas ciencias jurídicas sino también la de formar desde las necesidades profesionales: desde aquellas a las que han de atender las actividades de los juristas. O lo que es lo mismo, desde su trabajo profesional, lo que requiere conocimientos de varia entidad: oficina, marketing, gestión, uso de tecnologías, "informática jurídica", conocimientos sobre las leyes y las construcciones elaboradas al efecto: interpretación del Derecho, aplicación del Derecho, acceso al Derecho, ciencias del Derecho. Esto es: la formación debe ser desde las competencias profesionales que

\footnotetext{
${ }^{6}$ Vease, por ejemplo, los trabajos científicos publicados en las dos siguientes colecciones: SPRINGER. Law, Governance and Technology Series, 41 volúmenes, http://www.springer.com/series/8808?detailsPage=titles; Prensas Universitarias de Zaragoza, Legal Framework for the Information Society Series (LEFIS SERIES), 17 volúmenes, http://puz.unizar.es/colecciones/57/40-LEFIS+Series.html. También las publicaciones contenidas en las revistas: International Review of Law, Computers \& Technology, 32 volúmenes, https://www.tandfonline.com/toc/cirl20/current; European Journal of Law and Technology, 8 volúmenes, http://ejlt.org/index; Revista Democracia Digital e Governo Eletrônico, 8 volúmenes, http://buscalegis.ufsc.br/revistas/index.php/observatoriodoegov/index ...
} 
los juristas han de saber poner en práctica dados los objetivos y características de su respectiva profesión y no únicamente desde el conocimiento de los elementos fundamentales de la ciencia del derecho en sus diferentes variedades como ocurre desde la expansión de la tradición racionalista.

A continuación se muestra, por lo explicado en el Capítulo 3, la persistencia del interés en la discusión sobre "informática jurídica" (Capítulo 4). Ello se concreta utilizando dos ejemplos: la regulacion de las ciudades inteligentes y una breve presentación de las actividades que son precisas para poder hacer uso de datos abiertos. Estos ejemplos mostrarán que en el siglo XXI, dada la expansión de las las Tecnologías de la Información y la Comunicación (TIC), no sólo se ha de atender a la "informática" desde el punto de vista de las exigencias que tiene en el ejercicio profesional del jurista o las del "ingeniero informático" sino que se ha de atender a la misma por la propia implantación del desarrollo tecnológico, que requiere estar atentos a sus consecuencias sociales y preveer regulaciones en practicamente todos los campos de las actividades. No se trata de moderar o limitarlas, se trata de encauzar y en ocasiones potenciar y prevenir conociendo, mínimamente, las características de los principios, teorías y desarrollo de las técnicas informáticas. Como indicamos en el Capítulo 4: hay labores que no se pueden resolver tan sólo con conocimientos jurídicos. Existen labores que requieren inevitablemente interdisciplinariedad: conocimiento y práctica, competencias, sobre “informática jurídica”. Es decir se precisa la participación de expertos en los conocimientos y técnicas de modelización, reglas de programación, algoritmos y representación del conocimiento, necesarios para desarrollar aplicaciones a utilizar como auxilio de las actividades jurídicas, actividades que han de ser expresadas como ejercicio de competencias profesionales propias de juristas.

Al final (Capítulo 5) se responde a la pregunta que titula este trabajo.

\section{La dogmática: centro de los estudios de Derecho}

El punto de partida de la consideración de que la formación jurídica ha de centrarse en el estudio de los conceptos que integran el estudio del Derecho debe situarse en el Renacimiento, momento en el que se puso énfasis en Europa en que el objeto de conocimiento de la realidad está en la misma realidad: la naturaleza, la cual es conocida y ordenada conforme la muestran las categorías y métodos de la razón. Es decir la ciencia y sus procedimientos o métodos: inducción y deducción. Inducción en el caso del conocimiento empírico de la realidad diferente 
a fa humana: la naturaleza, según Bacon ${ }^{7}$. Deducción en el caso del conocimiento de las actividades del hombre según Descartes ${ }^{8}$, porque éste (el hombre) está caracterizado, como fundamentaría Kant, por la libertad ${ }^{9}$, cabiendo a partir de la misma organizar las construcciones racionales que, como la ciencia del Derecho o la dogmática, permiten su ejercicio en convivencia con el ejercicio de la libertad por otros hombres.

Con lo cual tanto en el ámbito de la naturaleza como en el del hombre se dieron pasos para establecer un conocimiento de la realidad alejado de las fuentes de conocimiento propias de la Edad Media: los textos revelados, especialmente la Biblia. Ello satisfacía los criterios de conocimiento expandidos en la época, y en el caso del Derecho ayudaba a la superación de la guerra de religiones que mantenían (siglo XVI) los gobernantes de la época al poder fundamentar el origen de su poder, y el del Derecho que establecían, en una instancia neutral como la razón, a cambio de la fundamentación en una u otra concepción de la religión: si católica o protestante, lo que generaba conflictos.

La fundamentación del poder en la razón no se hizo realidad ejemplar sino hasta finales del siglo XVIII a través de la Revolución: la francesa específicamente. Esta Revolución se basó en la práctica revolucionaria: el asalto al poder y el derrocamiento del Monarca, y en la teoría de la voluntad general, es decir el establecimiento del poder político en sus tres elementos contrabalanceados: legislativo, ejecutivo y judicial. Poder situado básicamente en la asamblea legislativa en la que estaban representados los integrantes de la Nación, que puede transformar en sus reuniones las voluntades, opiniones y creencias particulares en leyes ${ }^{10}$, así como en la Asamblea constituyente cuyos integrantes pueden crear la norma superior: la Constitución. De

\footnotetext{
${ }^{7}$ En (BACON, 2011). Es una significativa sección de esta obra, la titulada:"Descripción de una historia natural y experimental capaz de servir de base y fundamento a la verdadera filosofía".

8 “en lo que toca a la razón o al sentido, siendo, como es, la única cosa que nos hace hombres y nos distingue de los animales, quiero creer que está entera en cada uno de nosotros y seguimos en eso la común opinión de los filósofos" (DESCARTES, 2005, p. 69).

9 "Para saber lo que he de hacer para que mi querer sea moralmente bueno...basta preguntar: ¿puedes creer que tu máxima se convierta en ley universal” (KANT, 1932, p. 37).

${ }^{10}$ Preámbulo.- Declaración de los derechos del hombre y del ciudadano de 1789.- Especialmente: "Los representantes del pueblo constituidos en Asamblea nacional, considerando que la ignorancia, el olvido o el menosprecio de los derechos del hombre son las únicas causas de las calamidades públicas y de la corrupción de los gobiernos, han resuelto exponer, en una declaración solemne, los derechos naturales, inalienables y sagrados del hombre, a fin de que esta declaración, constantemente presente para todos los miembros del cuerpo social, les recuerde sin cesar sus derechos y sus deberes; a fin de que los actos del poder legislativo y del poder ejecutivo, al poder cotejarse a cada instante con la finalidad de toda institución política, sean más respetados y para que las reclamaciones de los ciudadanos, en adelante fundadas en principios simples e indiscutibles, redunden siempre en beneficio del mantenimiento de la Constitución y de la felicidad de todos." Luego sigue la descipción en artículos (17) de estos derechos. http://www.conseil-constitutionnel.fr/conseil-constitutionnel/francais/la-constitution/lesconstitutions-de-la-france/constitution-de-1791.5082.html (consultado el 11 de abril de 2018).
} 
hecho en Francia así ocurrió: la Asamblea creó la Constitución destinada a la fundamentación del poder en la voluntad general y a la expresión de la organización del poder político mediante el mecanismo de la separación de poderes, y los Códigos: especialmente el Código Civil. Código creado por los representantes de la voluntad general y que se entiende equivalente a la razón. Código que ha de ser considerado como única fuente de Derecho, para regir la vida diaria de los individuos, debiendo los juristas interpretarlo y aplicarlo en casos concretos ${ }^{11}$.

El afán de los protagonistas y gobernantes de la Revolución francesa de expandir sus “logros" por Europa no llegó a prosperar inicialmente al ser derrotado en su intento Napoleón Bonaparte. Pero el espíritu racionalista comenzaba a calar como necesidad requerida en la organización y el gobierno de una creciente sociedad industrial, liberal, que prohibía las asociaciones obreras y motivaba el funcionamiento del mercado, liberada la propiedad de las "manos muertas" mediante las expropiaciones y desamortizaciones de las clases del Antiguo Régimen, que se iba implantando en toda Europa a lo largo del siglo XIX. La expansión de la racionalidad jurídica tuvo lugar en el funcionamiento de regímenes políticos tradicionales: no revolucionarios. Las monarquías, que procedían del Antiguo Régimen, iban modificándose en sus sistemas jurídicos en acordanza con los principios del mercado además de mantenerlos en forma respetuosa con la tradición.

El autor que mejor supo conciliar tradición y modernidad fue en Prusia Savigny combinando de manera inteligente el Derecho romano, vigente, que se aplicaba en los tribunales y las reformas liberales. A estos efectos llegó a refundar el derecho romano clásico atendiendo a figuras jurídicas como: autonomía de la voluntad, derecho subjetivo, relación jurídica, Derecho como coacción o garantía externa de la libertad... ${ }^{12}$ Instituciones que eran necesarias para el establecimiento de la sociedad liberal en el marco de acción de unos regímenes políticos cuya justificación, pese a todo, seguía basándose en el principio de que el monarca era la representación de Dios en la tierra, como era el caso en Prusia.

Estas teorías fueron completadas a lo largo del siglo XIX por los fundadores de la dogmática, pandectística y la jurisprudencia de conceptos: (PUCHTA, 1838), (JHERING,

\footnotetext{
${ }^{11}$ El detalle sobre las leyes como fuente del derecho se recoge en el Título Preliminar: De la publicación, de los efectos y de la aplicación de las leyes en general. Arts. 1-6.1. Ver el texto en https://www.legifrance.gouv.fr/telecharger_pdf.do?cidTexte=LEGITEXT000006070721 (consultado el 11 de abril de 2018).

12 (SAVIGNY, 1840, parágrafos 4 a 16)
} 


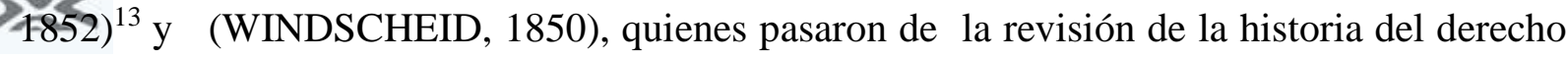
romano a su sustitución por los conceptos jurídicos “permanentes” desarrollo de los más básicos que serían los elementos del Código Civil alemán, cuya redacción comenzó en 1881, que entraría en vigor con el comienzo del siglo XX (el 1 de enero de 1900), tras la implantación desde varios años antes (desde 1871) del imperio alemán que continuaría como nación la legitimación de monarcas y señores anteriores de los territorios que lo constituyeron. Los tiempos habían cambiado y las sociedades se desarrollaban a partir del establecimiento de fábricas e industrias que generaban, desde comienzos del XIX, productos y cambios de vida propios del desarrollo económico de la época más que de la agricultura.

En España estos cambios tendrían repercusión en la segunda mitad del XIX. A comienzos del siglo ello todavía no se había introducido en la docencia. En concreto, en el plan de estudios de 12 de julio de 1807, el denominado Plan Caballero (Marqués de Caballero), el contenido del plan de derecho (PESET, 1968) y su espíritu quedaba recogido en el anuncio del resumen del mismo que se publicaría en la Gazeta de Madrid bajo el Gobierno de la Regencia de las Españas de 11/06/1813: "Guía para el estudio del derecho patrio, dividida en dos partes: la primera contiene las cilico tablas del resúmen del derecho español, que deben tomar de memoria los cursantes de Leyes en el quinto año de su estudio, en cumplimiento de lo mandado por la real cédula de 12 de julio de 1.807 sobre el nuevo plan de estudios en las universidades. La segunda comprehende el sumario de las 4040 leyes repartidas en los 340 títulos de los 12 libros de la novísima Recopilacion, publicada en 1805, y mandada pasar por los legistas en el séptimo y octavo año de su curso."14. El plan no aportaba novedades: exigía estudiar el elenco de las leyes vigentes desde hacía unos cuantos años sin atender a su clasificación en conceptos.

En otra línea, ya conceptual sin duda, estaría en España en la Ley de Instrucción Pública de 9 de septiembre de $1857^{15}$, la denominada Ley Moyano, que establecería pautas para todos los niveles de formación. En relación a la formación en Derecho establecía como materias más significativas, en su art. 43, las siguientes: Prolegómenos de Derecho, Historia é Instituciones del Derecho romano, Instituciones del Derecho civil, penal, mercantil, político, y administrativo de España, Economía política, Historia y ampliación del Derecho civil, penal y mercantil de

\footnotetext{
${ }^{13}$ Estudiamos el derecho romano "para inquirir el movimiento interno de la marcha del derecho, los ocultos resortes, las lejanas causas y la correlación inmaterial del conjunto del desenvolvimiento jurídico". (JHERING, 1852, pp.15-16).

${ }^{14}$ Gazeta de Madrid bajo el Gobierno de la Regencia de las Españas núm. 4, de 11/06/1813, página 44. PDF. Referencia BOE-C-1813-705.

15 Gazeta de Madrid, 10/09/1857
} 
España con el estudio de los Códigos y Fueros provinciales, Teoria y práctica de los procedimientos judiciales, Oratoria forense, Ampliación del Derecho administrativo en sus diversos ramos, Estadística, Derecho internacional común y particular de España, Legislación comparada. Puede comprobarse en los términos, por comparación al contenido del plan de 1807 antes mencionado que se centraba en el estudio de las leyes vigentes: Derecho romano o Derecho real, la aceptación preferente de las categorías de la desarrollada construcción conceptual que se estaba haciendo en diferentes zonas de la vida por la doctrina jurídica a lo largo del siglo XIX.

En el siguiente plan de estudios de Derecho de 1953, en vigor hasta tiempos recientes ${ }^{16}$, se quintaesenciaba las propuestas de carácter conceptual o dogmático al establecerse como materias contenidas en el mismo las siguientes: CURSO PRIMERO Derecho natural.- Historia e Instituciones del Derecho romano.- Historia del Derecho.- Derecho político.- Prácticas de lecturas de textos jurldicos clásicos (latinos y españoles). CURSO SEGUNDO Derecho político.- Derecho canónico.- Derecho civil (parte general).- Derecho penal (parte general).Economía política. CURSO TERCERO Derecho administrativo.-Derecho civil (obligaclones y contratos).- Derecho Internacional público.- Derecho penal (parte especial).- Hacienda pública. CURSO CUARTO Derecho administrativo (parte especial).- Derecho del trabajo.- Derecho civil (derechos reales e hlpotecario).- Hacienda pública (con especial atención al Derecho fiscal).- Derecho procesal.- Derecho mercantil. CURSO QUINTO Derecho civil (familia y sucesiones).- Derecho procesal.- Derecho mercantil.- Derecho internacional privado.- Filosofía del Derecho.

Una excepción. Efectivamente: en este plan (ya de 1953) se preveía una excepción a esta enseñanza dogmática. Ello fue, justamente, al mencionarse la Sociología en el siguiente Artículo de la norma. "Artículo undécimo.- Las Facultades de Derecho organizarán un curso de Sociología con especial referencia a los problemas jurídicos, que habrá de seguirse con carácter obligatorio, pero pudiendo elegir los alumnos el cursarlo en cualquiera de los años comprendidos entre el segundo y el quinto, ambos inclusive. Las Facultades organizarán igualmente Cursos prácticos de Contabilidad de interés habitual para el ejercicio de la profesión de Abogado. Estas prácticas de Contabilidad no serán obligatorias. pero a los alumnos que las

${ }^{16}$ BOE 29/08/1953, p. 5187. http://www.boe.es/datos/pdfs/BOE//1953/241/A05185-05190.pdf 
sigan con aprovechamiento se les expedirá un certificado o diploma de suficiencia en las mismas"17.

Este tipo de enseñanza articulaba conceptualmente, con absoluta preferencia, el estudio de las leyes: los Códigos y la doctrina dogmática que los estudiaba y reconstruía, lo que había sido hecho en Europa a lo largo del siglo XIX. Lo que era lógico porque éstas eran creadas por la razón expresada por el poder legislativo. Así en el Código civil español se establece un orden de prelación sobre las fuentes del Derecho según el cual la primera es la ley: “Artículo 1. 1. Las fuentes del ordenamiento jurídico español son la ley, la costumbre y los principios generales del derecho.”. Por otro lado, en el Real Decreto de 24 de julio de 1889 por el que se publica el Código Civil, éste quedaba ligado al poder legislativo: "Teniendo presente lo dispuesto en la ley de 26 de Mayo último; conformándome con lo propuesto por el Ministro de Gracia y Justicia, y de acuerdo con el parecer de mi Consejo de Ministros; En nombre de mi Augusto Hijo el Rey D. Alfonso XIII, y como Reina Regente del Reino, Vengo en decretar que se publique e inserte en la Gaceta de Madrid el adjunto texto de la nueva edición del Código Civil, hecha con las enmiendas y adiciones propuestas por la Sección de lo civil de la Comisión general de codificación, según el resultado de la discusión habida en ambos Cuerpos Colegisladores, y en cumplimiento de lo preceptuado por la mencionada ley de 26 de Mayo último. Dado en San Ildefonso a veinticuatro de Julio de mil ochocientos ochenta y nueve. MARÍA CRISTINA. El Ministro de Gracia y Justicia, JOSÉ CANALEJAS Y MÉNDEZ”18. Expresiones éstas que aun siendo coherentes con las propias del Antiguo Régimen así como la fundamentación teológica del poder, también centraban la responsabilidad de los textos en la voluntad y discusión de los legisladores conjuntamente con la Comisión general de codificación.

El cambio de funciones del jurista debido a la evolución del conocimiento: el paso de una socieda teológica a una sociedad racional, pero también al cambio del propio contexto social que era el paso de una sociedad caracterizada por el desarrollo de la agricultura y el mantenimiento de una sociedad dividida en estamentos, a una sociedad industrial, dividida en clases: burguesa y obrera, también se produjo en otros campos. Significativamente en el de la misma formación profesional que tenía lugar en todos momentos en la Universidad.

\footnotetext{
${ }^{17}$ Ibidem

18 Real Decreto de 24 de julio de 1889 por el que se publica el Código Civil, pp. 1-2. https://www.boe.es/legislacion/codigos/codigo.php?id=034_codigo_civil_y_legislacion_complementaria\&modo $=1$
} 
Pero ha habido que esperar porque el cambio se produjo a partir de los años setenta del siglo $\mathrm{XX}^{19}$. En la Universidad vigente en la época, heredera de la Universidad liberal de los siglos XVIII y XIX, la formación se entendía separada de las actividades de producción y servicios y la enseñanza se centraba en la adquisición de conocimientos disciplinares especializados: conceptuales, tal y como los hemos visto reflejados en anterioridad al mostrar los contenidos de la docencia impartida en la Facultad de Derecho. Se trataba de una Universidad encerrada en sí misma, que vivía en una auténtica torre de cristal racionalista, alejada de las demandas y necesidades de la Sociedad industrial guiada por el rápido desarrollo tecnológico en que estaba insertada. Ya exponíamos con anterioridad que los planes de estudio del Derecho estaban ligados a la expansión de los Códigos y la jurisprudencia de conceptos. Eran prácticamente idénticos los promulgados en España en 1857 y en 1953: en ambos casos el Derecho se aprendía por la acumulación de los contenidos de las dogmáticas, jurisprudencia de conceptos, propias de la respectiva época.

\section{Las competencias: el cambio hacia las necesidades profesionales}

En la década siguiente (la de los ochenta, siglo XX), la crisis económica y la fuerte competencia comercial hicieron que el planteamiento anterior quedase obsoleto y se legislaran reformas universitarias en diferentes países. Se insinuaba la necesidad de volver la mirada hacia la formación de los recursos humanos que las empresas necesitaban para subsistir en dicho entorno de competitividad. Esto requería de habilidades y competencias profesionales más allá de los conocimientos académicos y se reclamaba un cambio en el papel de la formación, que debía ser mucho más práctica.

Con ello la realidad de la Universidad se transformó desde la perspectiva curricular con la incorporación de conceptos como planificación-programación (basados en objetivos concretos a alcanzar a lo largo del tiempo), la introducción de ratios teoría-práctica (favoreciendo esta última: habilidades y competencias) y la incorporación de formas concretas de evaluación.

La concreción de este proceso ha tenido implantación en Europa y otros países a partir del denominado proceso de Bolonia, nombre con el que se expresan las iniciativas de cambio

\footnotetext{
${ }^{19}$ Sobre el cambio docente universitario puede verse (ESCANERO y otros, 2017, pp.51-62).
} 
adoptadas por los Ministros de Educación y las Universidades europeas surgidas a partir de la reunión a propósito que tuvo lugar en Bolonia en $1998^{20}$.

El proceso trata de armonizar (no homogeneizar, sino aumentar su compatibilidad y comparabilidad, respetando su diversidad) los sistemas universitarios europeos, de manera que todos ellos tengan una estructura homogénea de títulos de grado y postgrado. Con ello se pretende construir un espacio abierto en el que no existan obstáculos a la movilidad profesional de todos los universitarios.

Además. El cambio o mejora curricular presenta dos líneas de actuación de primordial importancia: a) el cambio de paradigma, que pasa a estar centrado en el aprendizaje o alumnado más que en la transmisión de la formación conceptual o erudita, y b) la introducción de las competencias.

Según la Declaración de Bolonia en lo referido a competencias el sistema de formación necesita centrarse en proporcionar una base consistente de competencias o requisitos (conocimientos, habilidades y actitudes) que se relacionan con el éxito profesional, personal y vital. Se precisa ese sistema a efectos de poder enmarcar no sólo la capacidad de innovar sino también la capacidad de adaptarse de forma rápida y eficaz a los cambios, utilizando y actualizando constantemente las competencias requeridas en la vida laboral.

Al mismo tiempo que la implantación de las reformas docentes, en conformidad con la reforma del Espacio Europeo de Enseñanza Superior, se ha producido en España en los últimos años un cambio notable en la formación de Abogados y Procuradores. El cambio es el que conlleva la aprobación de la Ley 34/2006 de 30 de octubre sobre el acceso a las profesiones de Abogado y Procurador de los Tribunales ${ }^{21}$. A continuación hacemos un breve resumen de la reforma.

El cambio es importante, además de por la implantación de los fines de la reforma educativa mostrados con anterioridad, por el hecho de que la Ley se ocupa de establecer el requisito de aprobar un examen de Estado para poder ejercer las profesiones citadas, frente a la normativa anterior que simplemente requería la obtención de la titulación de licenciado en Derecho: es decir la demostración del conocimiento básico de la jurisprudencia de conceptos en los diferentes campos desarrollados por la dogmática o ciencia del Derecho, para ser admitido en la práctica profesional por los respectivos Colegios profesionales.

\footnotetext{
${ }^{20}$ Ibidem.

${ }^{21}$ El texto de la ley se encuentra en: https://www.boe.es/buscar/act.php?id=BOE-A-2006-18870.
} 
Es por ello que en España se ha hecho necesaria la reforma de los estudios universitarios dirigidos a la formación de juristas, por ahora la de Abogados y Procuradores, estableciendo como objetivo de los mismos, tanto en la docencia de grado como en la concreta de postgrado (Master) ordenada para cada profesión, dotar a los futuros juristas del conjunto de competencias profesionales que les son precisas para ejercer las actividades jurídicas ${ }^{22}$.

Estas competencias profesionales de los juristas han sido fijadas en la nueva regulación mediante la consideración de cuál se entiende es en la actualidad el ejercicio de una práctica acomodada a las condiciones culturales, sociales, económicas y geográficas en las que los juristas las ejercen. Competencias que en el Estado de Derecho han quedado establecidas tanto en el contenido de las Leyes que establecen genéricamente los procedimientos destinados a la promulgación y la aplicación del Derecho como en las características de estas actividades propias de juristas.

De esta forma la Ley que regula el acceso a la profesión de abogado y procurador, Ley 34/2006, de 30 de octubre, sobre el acceso a las profesiones de Abogado y Procurador de los Tribunales, define al abogado, en su artículo 1.1, como colaborador "en el ejercicio del derecho fundamental a la tutela judicial efectiva, con el fin de garantizar el acceso de los ciudadanos a un asesoramiento, defensa jurídica y representación técnica de calidad”. La definición se concreta en actividades y ejercicio profesional en mayor medida en el art. 1.2: "La obtención del título profesional de abogado en la forma determinada por esta ley es necesaria para el desempeño de la asistencia letrada en aquellos procesos judiciales y extrajudiciales en los que la normativa vigente imponga o faculte la intervención de abogado, y, en todo caso, para prestar asistencia letrada o asesoramiento en Derecho utilizando la denominación de abogado; todo ello sin perjuicio del cumplimiento de cualesquiera otros requisitos exigidos por la normativa vigente para el ejercicio de la abogacía”.

Las cualificaciones profesionales requeridas han quedado especificadas en el Reglamento que concreta la Ley: el Real Decreto 775/2011, de 3 de junio, por el que se aprueba el Reglamento de la Ley 34/2006, de 30 de octubre, sobre el acceso a las profesiones de Abogado y Procurador de los Tribunales ${ }^{23}$. Esta norma regula el contenido del aprendizaje que han de realizar dichos profesionales, antes y después de la obtención de un Diploma de Grado en

\footnotetext{
${ }^{22}$ Sobre la necesidad de que el curriculum académico dogmático "clásico" de las Facultades de Derecho "continentales" se modifique adaptándolo a competencias o formación práctica existen referencias extensas al caso alemán en: (PILNIOK, BROCKMANN, 2017).

${ }^{23}$ El texto se encuentra en: https://www.boe.es/diario_boe/txt.php?id=BOE-A-2011-10459.
} 
Berecho o de un título de Master en abogacía, tanto mediante la participación en cursos impartidos por profesores universitarios y abogados, cuanto que por la realización de ejercicios en el periodo de formación práctica que debe tener lugar en instituciones donde se realizan las actividades jurídicas profesionales.

Como puede comprobarse a continuación tanto las competencias establecidas para el Grado como las referidas a los estudios de postgrado están referidas a las que las teorías explican, presentan o expresan la realidad jurídica en forma de actividades profesionales.

A continuación relacionamos las competencias para ser abogado con las actividades típicas del profesional del Derecho:

a) Actividades jurídicas propiamente dichas:

- dominar los conceptos abstractos, dogmática o ciencia del derecho, por medio de los que se conoce el ordenamiento, el marco jurídico nacional e internacional y los distintos ámbitos de la práctica profesional,

- interpretar el Derecho atendiendo a la regulación de la práctica judicial y la caracterización de los problemas,

- aplicar el Derecho en forma judicial y extrajudicial.

Estas competencias dan cuenta de cómo el curriculum académico del Grado de Derecho, también, pero en menor medida, el curriculum de Master en abogacía, mantiene las asignaturas, con escasos cambios de denominaciones, que la ciencia del derecho o dogmática ha ido elaborando desde que los Códigos se desarrollaron e implantaron a finales del XVIII, a lo largo del siglo XIX. Especialmente al expresar que los juristas deben "dominar los conceptos abstractos, dogmática o ciencia del derecho, por medio de los que se conoce el ordenamiento, el marco jurídico nacional e internacional y los distintos ámbitos de la práctica profesional". ${ }^{24}$

A continuación vemos cómo, en cambio, el resto de las competencias están alejadas de los "conceptos abstractos":

b) Actividades profesionales de gestión, conformes con reglas éticas y administrativas propias de la organización y puesta en funcionamiento de oficinas, negocios o empresas.

Estas son competencias relacionadas con el funcionamiento de una oficina profesional.

\footnotetext{
${ }^{24}$ Ello puede verse en las asignaturas del Grado de Derecho de la Facultad de Derecho de Zaragoza: denominaciones que se encuentran en: https://estudios.unizar.es/estudio/ver?id=132 (consultado el 11 de abril de 2018).También, por ejemplo, en las que se imparten en la Licenciatura de la Facultad de Derecho de la Universidad de Lisboa: http://www.fd.ulisboa.pt/cursos/licenciatura/plano-de-estudos/ (consultado el 11 de abril de 2018). En general las Facultades de Derecho de tipo continental son conservadoras en lo relativo al cambio de curriculum.
} 
c) Actividades de ejercicio y uso de herramientas propias de la "sociedad del conocimiento" en la que vivimos.

La referencia es aquí a las tecnologías de la información y la comunicación (TIC), de uso indispensables en cualquier actividad profesional en la actualidad. Aquí podemos incluir a las actividades de las que se ocupa la "informática jurídica" como decíamos al principio.

d) Actividades comunicativas propiamente dichas: dirigidas al logro y mantenimiento del diálogo y el consenso, así como a poner en práctica las ventajas del trabajo en equipos interdisciplinares.

Competencias, las últimas (las incluidas en b), c) d)), que van más allá de los conocimientos de los argumentos dogmáticos. Más bien están referidas al respecto a la pluralidad cultural, única forma de mantener el diálogo y el consenso ${ }^{25}$, y, finalmente...

e) Actividades orientadas por la puesta en acción de los principios de justicia o de salvaguarda de los derechos humanos en el Estado de Derecho tal y como se concreta en los siguientes textos descriptivos de las competencias a las que se hace referencia en el art. 10 del Reglamento tomado en consideración:

- “evitar situaciones de lesión, riesgo o conflicto en relación a los intereses encomendados o su ejercicio profesional ante tribunales o autoridades públicas y en las funciones de asesoramiento",

- "conocer las diferentes técnicas de composición de intereses...",

- "conocer y saber aplicar los derechos y deberes deontológicos profesionales...",

- "conocer y evaluar las distintas responsabilidades vinculadas al ejercicio de la actividad profesional, incluyendo el funcionamiento básico de la asistencia jurídica gratuita y la promoción de la responsabilidad social del abogado",

- "saber identificar conflictos de intereses y conocer las técnicas para su resolución, establecer el alcance del secreto profesional y de la confidencialidad, y preservar la independencia de criterio",

- “desarrollar destrezas y habilidades para la elección de la estrategia correcta para la defensa de los derechos de los clientes...",

- "saber desarrollar destrezas que permitan al abogado mejorar la eficiencia de su trabajo y potenciar el funcionamiento global del equipo o institución en que lo desarrolla...",

\footnotetext{
${ }^{25}$ Sobre la antigua y reciente historia, así como su complejidad y relevancia, de las teorías de la argumentación, claves en una formación jurídica, ver: (BRETON, GAUTHIER, 2011).
} 
“...extraer argumentalmente consecuencias jurídicas, en atención al contexto y al destinatario al que vayan dirigidas, de acuerdo en su caso con las modalidades propias de cada ámbito procedimental”, y

- "saber desarrollar habilidades y destrezas interpersonales, que faciliten el ejercicio de la profesión de abogado en sus relaciones con...”.

Estas competencias están relacionadas con principios generales del Derecho, justicia, valores... que hoy se concretan en las Declaraciones de Derechos Humanos de los que se ocupan entidades estatales, internacionales y organizaciones no gubernamentales.

Como es natural, lo que ya se mencionaba con anterioridad, este detallado entorno competencial es tomado como referencia internacionalmente en unos casos y en otros no. Ello depende de la tradición y particularidades de la formación jurídica existente en cada país ${ }^{26}$.

\section{Informática jurídica}

Aquí nos vamos a fijar, a partir de experiencias desarrolladas o en desarrollo, en las siguientes manifestaciones de "informática jurídica": 1) la necesidad de regular un Derecho dirigido a considerar los efectos de la introducción de los programas de ordenador en las actividades humanas, y 2) las competencias profesionales necesarias para el trabajo interdisciplinar que integra la expresión "informática jurídica”..

Para lo primero partimos de lo desarrollado en una experiencia realizada entre 2010 y $2014^{27}$ en la elaboración de propuestas jurídicas para el diseño y construcción técnica de “ciudades inteligentes". Para lo segundo de la reseña de los pasos básicos que se están dando para construir un servicio concreto, modélico, de utilización de "datos abiertos".

\footnotetext{
${ }^{26}$ La página web http://www.teachinglegalethics.org/ es la página propia del denominado "International Forum on Teaching Legal Ethics and Professionalism". En la página pueden verse distintos ejemplos del grado de expansión internacional sobre competencias profesionales en el ámbito continental y en el de derecho de "common law".

${ }^{27}$ Se trataba del proyecto Ciudad 2020 - Hacia un nuevo modelo de ciudad inteligente sostenible. El proyecto pretendía lograr un avance en las áreas de eficiencia energética, Internet del futuro, Internet de las cosas, comportamiento humano, sostenibilidad medioambiental y movilidad y transporte, con el objetivo de diseñar la ciudad del futuro, sostenible, inteligente y eficiente. Hay referencias en: https://www.indracompany.com/es/indra/ciudad-2020-modelo-ciudad-inteligente-sostenible (consultado el $11 \mathrm{de}$ abril de 2018).
} 


\section{1.- Ciudades inteligentes ${ }^{28}$}

Por supuesto que, tras el adecuado conocimiento de los servicios y las infraestructuras técnicas (informáticas, estadísticas, sensores) precisos para formar ciudades inteligentes aportados por técnicos expertos, y el dominio de las competencias precisas para ello, la principal necesidad es la de que se construyan respetando los derechos de los ciudadanos recogidos en la Constitución y la totalidad de las normas que integran el ordenamiento, que en la actualidad están muy desarrolladas en conformidad con lo que precisa el uso de las TIC en la vida diaria. Esto implica requerir que entre los elementos de conocimiento con los que se cuente a la hora de diseñar los servicios de las futuras ciudades inteligentes estén presentes los aspectos jurídicos $\mathrm{o}$, dicho resumidamente, el contenido del ordenamiento o, más acertadamente, las competencias exigidas a los abogados para serlo como mencionábamos con anterioridad. Este es un requisito imprescindible a la hora del diseño, si atendemos a que con los servicios previstos se esperan cambios sociales de relevancia que afectan a los derechos adquiridos por parte de quienes los prestan y a los derechos de los titulares de la información, los ciudadanos, con la que se construyen dichos servicios ${ }^{29}$.

Ello significa que si atendemos, por ejemplo, a que si en la construcción de los servicios o programas se ha de contar con información captada por sensores de cambios ocurridos o por ocurrir en la naturaleza, su uso ha de estar permitido por quienes la proporcionen porque son poseedores de los sensores y de los análisis e interpretaciones de datos que los sensores capturan y facilitan a efectos de construir la información. Los propietarios de los sensores y los que realicen las interpretaciones de los datos deberán, además, asumir la responsabilidad por la calidad de la información y por las consecuencias de efectos no previsibles ocasionados por los programas que procesen dicha información. Es decir han de participar en la construcción de los servicios personas que dominen competencias técnicas y personas que dominen competencias jurídicas.

\footnotetext{
${ }^{28}$ El presente apartado resume el trabajo (GALINDO, 2018).

${ }^{29}$ Tengamos en cuenta que la mencionada plataforma tecnológica Ciudad 2.020 tomada como referencia podrá proveer servicios a los ciudadanos que si en la actualidad son prestados, por ejemplo, por empleados de organizaciones como pueden ser agencias de viaje, en el futuro lo serán por programas de ordenador, propiedad de empresas, que facilitarán siguiendo los protocolos adecuados a los interesados reservas de billetes de avión o tren, alojamiento y medios de transporte (alquiler de automóviles) en general. Esos programas o servicios son los que producirán la conexión precisa entre los ciudadanos, los clientes, y los proveedores. Este ejemplo, que como se sabe es una realidad que ha obligado a transformar el funcionamiento de las agencias de viaje, es una, pequeña, señal de lo que se persigue en los proyectos de construcción de servicios destinados al funcionamiento de las ciudades inteligentes.
} 
Si la información está referida a las actividades de la vida diaria, es decir a la identificación personal y a los datos de la vida de los ciudadanos, su uso ha de ser voluntariamente consentido por éstos, siendo preferible que la utilización de los datos personales se haga transformándolos en formato anónimo a efectos de preservar en la mayor medida de lo posible el derecho de cada ciudadano a la intimidad de su vida personal reconocida por la Constitución, aplicando de esta forma lo que prescriben las medidas de seguridad que están reconocidas como parte del ordenamiento jurídico en esta materia. De esta forma todo tipo de información podrá ser tratado atendiendo a pautas de comportamiento individuales, de usuarios de los servicios, pudiendo ponerse a disposición de quien las ha generado o a la de otros usuarios/ciudadanos que los adquieran. La responsabilidad, igualmente, quedará acotada a las de las empresas que creen o mantengan los servicios.

A nadie se le puede escapar la trascendencia social de los servicios/aplicaciones que se están diseñando y poniendo en práctica con vistas a alcanzar el objetivo final de la implantación de la ciudad inteligente. Se trata de transformar la prestación de servicios realizada hasta ahora por empresas, organizaciones y personas concretas contando con su especialización profesional, en la prestación de otros servicios elaborados y suministrados de forma automática a partir de los datos "sensados" por autómatas y lo experimentado previamente por las mismas $\mathrm{u}$ otras personas ${ }^{30}$.

La cuestión que hay que resolver es ¿cómo han de ser diseñados los programas/servicios/ autómatas/"artefactos" que integran las ciudades inteligentes a efectos de poder satisfacer con ellos las necesidades para las que se elaboran a la vez que la preservación de los derechos y deberes propios de todos aquellos que participan en el proceso de diseño, suministro, adquisición y uso de dichos servicios/programas que están garantizados por la normativa propia de los sistemas jurídicos democráticos?

Para responder a lo anterior hay que tener en cuenta que son tres los elementos necesarios para la construcción de los programas:

1. La construcción de bancos de datos o el diseño de los programas.

2. La comunicación entre usuarios y bancos de datos.

3. Las prescripciones de la normativa vigente para la construcción y uso de los programas/servicios.

\footnotetext{
${ }^{30}$ Estamos ante la introducción generalizada de robots más allá del ámbito militar o la construcción de automóviles. Ver algunas consecuencias desde una perspectiva jurídica en (PAGALLLO, 2013).
} 
A continuación detallamos algunas de las características del último elemento: el relativo a la regulación jurídica o a las competencias jurídicas. Los dos primeros elementos requieren, en cambio, considerar elementos tecnológicos: competencias propias de ingenieros informáticos.

Cuando hablamos sobre la regulación jurídica de los sistemas/programas de las ciudades inteligentes se produce la paradoja de que hablamos de algo desconocido porque que no existe regulación jurídica sobre Ciudades Inteligentes fuera de la normativa que potencia la realización de investigaciones y desarrollos sobre las mismas ${ }^{31}$, o los acuerdos del mismo carácter pactados entre las organizaciones administrativas, ayuntamientos fundamentalmente, que encargan el diseño de programas y servicios para los ciudadanos en razón a su obligación genérica de apoyar el I+D+i y su capacidad de crear empleo ${ }^{32}$.

La verdad es que no es fácil avanzar jurídicamente según las pautas propias de las teorías del Derecho positivistas: aquéllas que se limitan a hacer una exégesis de la Ley. Para estas teorías, y la dogmática, la ciencia del derecho, si no existen leyes el jurista no puede realizar consideraciones jurídicas: si las hace estaría haciendo política únicamente y éste es un cometido propio del legislador que es quien hace las leyes. En cambio para otras teorías jurídicas como las comunicativas que se ocupan, como cometido propio de su competencia profesional, de estudiar actividades y su acomodación o no a las normas, principios y valores de una sociedad democrática vigentes, no existen tales dificultades ${ }^{33}$, porque desde esta última perspectiva no sólo cabe sino que, al contrario, es preciso realizar manifestaciones sobre la normativa a la que ha de atenderse en el diseño e implantación de los programas servicios inteligentes, dado el relevante carácter y la importante función social de sus servicios y los requisitos legales/valorativos que éstos precisan satisfacer aun cuando se esté hablando per ahøra de un fenómeno general limitado, por ahora, al ámbito de I+D+i. También hay que decir que la

\footnotetext{
${ }^{31}$ Existe en España un Comité técnico (AEN/CTN 178 - Ciudades inteligentes) para el desarrollo de estándares industriales para ciudades inteligentes: que ha creado varios estándares al respecto: http://www.aenor.es/aenor/normas/ctn/fichactn.asp?codigonorm=AEN/CTN\%20178\#.UsFCRdLuKSo. (consultado el 11 de abril de 2018). Existe también en ISO el Comité ISO/TC 268 dedicado al desarrollo sostenible en comunidades: http://www.iso.org/iso/iso_technical_committee?commid=656906 (consultado el 11 de abril de 2018).

${ }^{32}$ Este es el caso de "smartcity" "La red española de Ciudades Inteligentes". Ver la página web en: http://www.redciudadesinteligentes.es (consultado el 11 de abril de 2018). En el art. 3 de los Estatutos se establece que el objeto de la red es: "La Asociación tiene por objeto la generación de una dinámica entre ciudades con el finde disponer de una 'red Española de ciudades inteligentes' las cuales han de promover la gestión automática y eficientede las infraestructuras y los servicios urbanos, así como la reducción del gasto público y la mejora de la calidadde los servicios, consiguiendo de este modo atraer la actividad económica y generando progreso"

${ }^{33}$ Ver al respecto (GALINDO, 1998). Otras posiciones establecen la conexión existente entre aspectos jurídicos y gobernanza, otra forma de promover el estudio interdisciplinar del Derecho: (DOUGLAS-SCOTT, 2013).
} 
perspectiva jurídica ha de adoptarse desde el momento de diseño de los sensores y los artefactos o programas porque de otra forma los servicios/sistemas no podrían ser utilizados en el futuro debido a la manifiesta ilegalidad en la que incurrirían quienes los diseñaran y utilizaran en el caso de que los sistemas generaran servicios defectuosos ${ }^{34}$.

La propuesta no es novedosa: la implantación del comercio y el gobierno electrónicos requirió también el establecimiento previo de normas jurídicas que permitieron el funcionamiento de los programas/sistemas en conformidad con las reglas y principios de los ordenamientos jurídicos con el fin de superar las limitaciones encontradas en el uso de las tecnologías. A lo anterior se añade la obvia consideración de que la normativa a la que también hay que atender en el diseño de servicios para las ciudades inteligentes es la existente para regular el funcionamiento de la sociedad de las TICs desde hace ya cierto tiempo (los años setenta del siglo XX): protección de datos personales, medidas de seguridad, firma electrónica, acceso electrónico de los ciudadanos a los servicios públicos, preservación de la propiedad intelectual e industrial y las medidas generales del ordenamiento jurídico previstas para la preservación y atribución de responsabilidad. Esto es informática jurídica.

Todo lo anterior expresa que los proveedores de servicios inteligentes deben hacer unas claras previsiones sobre responsabilidades del uso de sus servicios y recogerlas en los contratos a realizar con quienes dan los datos y con quienes utilizan sus servicios ${ }^{35}$. De todas formas no ha de quedar duda de que estas actividades son propias de la "informática jurídica": tienen carácter interdisciplinar, en ellas han de actuar técnicos y juristas, es preciso saber desenvolverse en el ámbito competencial de ambos campos profesionales.

\section{2.- Datos abiertos}

Otro ejemplo actual de exigencia del trabajo interdisciplinar es el constituido por la utlización de los “datos abiertos” de carácter público y privado que se ofrecen en Internet. Este es un próximo paso a dar por el desarrollo tecnológico, demandado por el estado de la técnica y las propias normas que ya facilitan el acceso a datos e intentan poner en realidad la transparencia de las actividades de las instituciones públicas ${ }^{36}$.

\footnotetext{
${ }^{34}$ Es el caso de lo que sucede con Facebook y las aplicaciones diseñadas por Cambridge Analitycs puestas en cuestión por su abuso de la información personal. Ver noticia explicativa al respecto de 1 de marzo de 2018 en: http://www.bbc.com/mundo/noticias-43226416 (consultado el 11 de abril de 2018).

35 Ver la relevancia de esta clase de contratos en (OSSOWSKI, 2013).

${ }^{36}$ Se recogen ejemplarmente estas necesidades en: (MEHR 2017)
} 
Estos datos están publicados en Internet normalmente bajo la legitimidad que les da el hecho de que una empresa o una Administración o una institución los hacen públicos satisfaciendo los correspondientes Derechos que ostentan sobre los mismos los que los publican o sus propietarios que se lo permiten. Al mismo tiempo son precisos juristas que han de valorar la legalidad del uso de la información almacenada y recopilada. Con lo cual ha llegado el tiempo de su utilización por el desarrollo de aplicaciones informáticas que los procuren a quienes tienen necesidad de los mismos ${ }^{37}$.

Aquí hay que decir que el desarrollo de estas aplicaciones, como ocurre en el caso de las ciudades inteligentes, sólo es posible con la intervención de los técnicos que saben modelizar la información y las peticiones de la misma hecha a través de aplicaciones desarrolladas a propósito. Este es otro ejemplo claro de necesidad del trabajo interdisciplinar propio de la "informática jurídica" 38 .

\section{Conclusiones}

La informática jurídica es necesaria en 2018. Pero no lo es porque sea una ciencia del derecho o una especialidad informática. Tampoco porque haya enseñanza de la misma, o se intente construir una dogmática, contando con los conceptos que organiza. Su razón es mucho más concreta: es obra de la satisfacción de necesidades profesionales, como lo son la regulación de las TIC y la elaboración de programas de ordenador, labores que para completarlas adecuadamente han de realizarse interdisciplinarmente: mediante los conocimientos, técnicas, teorías, prácticas y usos que propone la informática jurídica. Es decir es obra del adiestramiento en el ejercicio de competencias propias de los juristas y en el de competencias propias de los ingenieros informáticos.

\section{REFERENCIAS}

\footnotetext{
${ }^{37}$ Sobre datos abiertos y "big data" y sus implicaciones jurídicas y sociales ver (HOEREN, KOLANY- RAISER, 2018).

${ }^{38}$ Esta es, resumidamente, la metodología que se está siguiendo por el Grupo de Investigación sobre Protección de datos, Documentación y Multiculturalismo de la Universidad de Zaragoza, en la elaboración de un programa de acceso al Camino de Santiago utilizando datos abiertos.
} 
BACON, F., La Gran Restauración (Novum Organum), Tecnos, Madrid, 2011.

BRETON, P., GAUTHIER, G., Histoire des Théories de l'argumentation, La Découverte, Paris, 2011.

DESCARTES, R., Discurso del método y Meditaciones metafísicas,Tecnos, Madrid, 2005 .

DOUGLAS-SCOTT, S., Law after Modernity, Hart Publishing Ltd, Oxford, 2013.

ESCANERO, F., GALINDO, F., GUERRA. M., LASALA, P. SORIA,M.S., Estudio sobre el aprendizaje. A partir de varias experiencias realizadas en Facultades de Medicina y Dereho, Prensas de la Universidad de Zaragoza, Zaragoza, 2017.

GALINDO, F., The communicative concept of law. Journal of legal pluralism and unofficial law, Vol 41, 1998, pp. 111-129.

GALINDO, F., Ciudades inteligentes y derechos del ciudadano, publicado en: LUCAS MARTÍN. F.J, VIDAL GIL, E.J., FERNÁNDEZ RUIZ-GÁLVEZ, E., BELLVER CAPELLA, V., (coords.) Pensar el tiempo presente, Tirant lo Blanch, Valencia, vol. 1, 2018, pp. 641-662.

GRANT, D., BENNET MOSSES, L., Technology and the trajectory of myth, Elgar, Chettenham, 2017.

HOEREN. T., KOLANY- RAISER, B., (eds.), Big Data in Context. Legal, Social and Technological Insights, Springer, Cham, 2018.

JHERING, R.v., Geist des römischen Rechts auf den verschiedenen Stufen seiner Entwicklung. Teil 1. Leipzig, 1852, Scientia Verlag, Aalen, 1968, vol. 1. 
KANT, I., Fundamentación de la metafísica de las costumbres, Espasa Calpe, Madrid, 1932.

MEHR, H., Artificial Intelligence for Citizen Services and Government, Harvard Ash $\begin{array}{lllll}\text { Center Technology \& } \quad \& \quad \text { Democracy } & \text { Fellow, August }\end{array}$ (https://ash.harvard.edu/files/ash/files/artificial_intelligence_for_citizen_services.pdf, (consultado el 11 de abril de 2018))

OSSOWSKI, S., Agreement Technologies, Springer, Dordrecht, 2013.

PAGALLO, U., The Laws of Robots. Crimes, Contracts, and Torts, Dordrecht, Springer, 2013.

PALIWALA, A., (ed.) A history of legal informatics, Prensas Universitarias, Zaragoza, 2010 .

PESET, M, La enseñanza del derecho y la legislación sobre Universidades, durante el reinado de Fernando VII (1808-1833), en Anuario de Historia del Derecho Español, 1968, pp. 238-243.

PILNIOK, A., BROCKMANN, J., (eds.), Die juristische Profession und das Jurastudium, Nomos, Baden-Baden, 2017.

PUCHTA, G.F., Lehrbuch der Pandekten, Verlag von Johann Ambrosius Barth, Leipzig, 1838.

SAVIGNY F.K., System des heutigen Römischen Rechts, vol. 1, Veit, Berlin, 1840.

WINDSCHEID, B., Die Lehre des römishen Rechts von der Voraussetzung, Julius Buddeus, Düseldorf, 1850. 